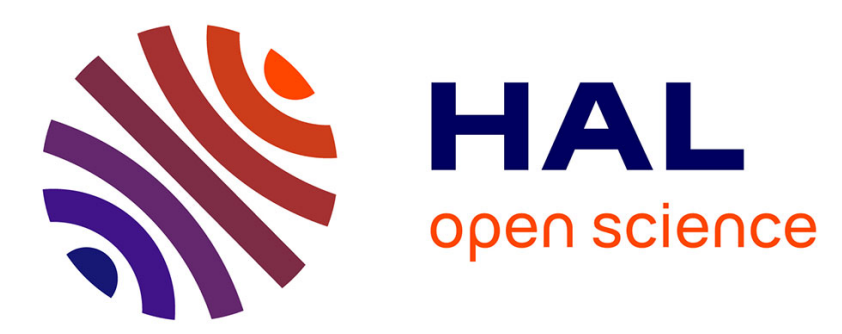

\title{
Fine Structure of Diffuse Scattering Rings in Al-Li-Cu Quasicrystal: A Comparative X-ray and Electron Diffraction Study
}

\author{
P. Donnadieu, F. Dénoyer
}

\section{To cite this version:}

P. Donnadieu, F. Dénoyer. Fine Structure of Diffuse Scattering Rings in Al-Li-Cu Quasicrystal: A Comparative X-ray and Electron Diffraction Study. Journal de Physique I, 1996, 6 (11), pp.1477-1485. 10.1051/jp1:1996159 . jpa-00247260

HAL Id: jpa-00247260 https://hal.science/jpa-00247260

Submitted on 1 Jan 1996

HAL is a multi-disciplinary open access archive for the deposit and dissemination of scientific research documents, whether they are published or not. The documents may come from teaching and research institutions in France or abroad, or from public or private research centers.
L'archive ouverte pluridisciplinaire HAL, est destinée au dépôt et à la diffusion de documents scientifiques de niveau recherche, publiés ou non, émanant des établissements d'enseignement et de recherche français ou étrangers, des laboratoires publics ou privés. 


\title{
Fine Structure of Diffuse Scattering Rings in Al-Li-Cu Quasicrystal: A Comparative X-ray and Electron Diffraction Study
}

\author{
P. Donnadieu $\left({ }^{1, *}\right)$ and F. Dénoyer $\left({ }^{2}\right)$ \\ $\left({ }^{1}\right)$ Laboratoire d'Etude des Microstructures $\left({ }^{* *}\right)$, BP 72, 92322 Châtillon Cedex, France \\ $\left({ }^{2}\right)$ Laboratoire de Physique des Solides ( ${ }^{* * *}$ ), Bâtiment 510, Université Paris XI, \\ 91405 Orsay Cedex, France
}

(Received 11 December 1995, revised 10 April 1996, accepted 26 July 1996)

PACS.61.44.Br - Quasicrystals

\begin{abstract}
A comparative X-ray and electron diffraction study has been performed on Al$\mathrm{Li}-\mathrm{Cu}$ icosahedral quasicrystal in order to investigate the diffuse scattering rings revealed by a previous work. Electron diffraction confirms the existence of rings but shows that the rings have a fine structure. The diffuse aspect on the X-ray diffraction patterns is then due to an averaging effect. Recent simulations based on the model of canonical cells related to the icosahedral packing give diffractions patterns in agreement with this fine structure effect.
\end{abstract}

Résumé. - Nous comparons les diagrammes de diffraction des rayons-X et des électrons obtenus sur les mêmes échantillons du quasicristal icosaèdrique Al-Li-Cu. Notre but est d'étudier les anneaux de diffusion diffuse mıs en évidence par un travail précédent. Les diagrammes de diffraction électronıque confirment la présence des anneaux mais ils montrent aussı que ces anneaux possèdent une structure fine. L'aspect diffus des anneaux révélés par la diffraction des rayons X est dû à un effet de moyenne. Des simulations récentes basées sur la décomposition en cellules canonıques de l'emplement icosaédrique produisent des diagrammes de diffraction en accord avec ces effects de structure fine.

Since the discovery of the Al-Mn icosahedral quasicrystal, the origin of the stability of this state remains a matter of debate. First, quasicrystalline phases were considered as metastable phase because they were obtained by rapid solidification. But this opinion has been soon reexamined with the elaboration of the $\mathrm{Al}_{6} \mathrm{Li}_{3} \mathrm{Cu}$ quasicrystal by conventional solidification [1]. Later on, the stability has been strongly established in the AlCuFe and AlPdMn quasicrystal alloys [2,3]. These phases which are obtained by conventional solidification achieve perfect quasicrystallinity after annealing at high temperature; i.e. narrow diffraction line widths and no deviation to icosahedral symmetry [4].

Two points of view have been developed to explain this stability. The former considers that a large cohesion energy due to the quasicrystal electronic structure is responsible for the stability [5]. The later points out that an entropy stabilization is possible; this is based on

(*) Author for correspondence (e-mail: donnad@onera.fr)

$\left({ }^{* *}\right)$ UMR 104 CNRS/ONERA

$\left({ }^{* * *}\right)^{\prime}$ URA 2 CNRS

(C) Les Éditions de Physıque 1996 
the properties of random tilings in which a high degree of disorder can be introduced without strongly altering the quasicrystalline state. In that case, disorder is expected to generate diffuse wings at the Bragg peaks [6]. Therefore, a considerable interest is now given to diffuse scattering in quasicrystal.

In fact interest has started before the debate on the stability because soon after the discovery of Al-Mn quasicrystals, diffuse scattering rings or arcs have been mentioned [7]. These diffuse rings seems now to be a quite general feature. They have been observed in numerous phases obtained by rapid as well as conventional solidification (Al- $\mathrm{Li}-\mathrm{Cu}[8,9], \mathrm{Al}-\mathrm{Cr}$ [10], Ti-Mn [11], Ti-Transition Metal alloys [12]. The diffuse rings shows the same characteristics: they are observable of the 2 -fold diffraction patterns and absent on the 3 -fold and 5-fold patterns; they are centered on positions of a I-type reciprocal lattice which are forbidden for the quasicrystal primitive lattice.

Several attempts have been made to understand diffuse scattering within the framework of the icosahedral glass model. This model depicts the quasicrystal as a distribution of icosahedral clusters, these clusters being linked to each other in a way that preserve long range orientational order. It was expected that this icosahedral glass provide the answer since topological or chemical disorder may easily be generated in such a glass. Disorder can arise from several aspects: - the multiplicity of the linkage configurations between icosahedral clusters - the existence of several types of clusters within the glass - the differences between the glue atoms and the atoms involved in the icosahedral clusters. The ring positions suggest the possibility of a short range order-long range order transition from a P-type to a F-type quasicrystal but such transition has been only observed in one case [13].

Goldman et al. [9] attributed the disorder to the multiplicity of the two-cluster configurations. The diffuse scattering arises then from the average on all the possible configurations. They obtained diffuse rings in qualitative agreement with the observations without introducing a particular cluster decoration. Another attempt to understand diffuse scattering using the icosahedral glass model has been applied to the Ti-Mn quasicrystals. These quasicrystals exhibit very pronounced diffuse scattering which allows for a deeper investigation and better comparıson with the model. According to Gibbons et al. [11], only partial agreement was found between the diffuse rings and the icosahedral glass model predictions and modification of the cluster decoration did not give a better match. Gibbons and Kelton [14] have also considered the influence of constrained first neighbour distances in the icosahedral cluster. They prescribe a good local environment which leads to a sharpening of peaks and diffuse arcs on the calculated diffraction patterns.

Transition-state models are able to give diffuse scattering for the states of chemical order intermediate between long range and short range order [15]. However, when applied to the case of Ti-Mn quasıcrystals, the transition-state model accounts for only a part of the diffuse arcs [11]. A detailed study of composition and heat treatment effects on the Ti-Mn quasicrystal diffuse arcs has been performed by Kelton and Gibbons [12]. Diffuse features appear unsensitive to annealing and cooling conditions. Partial substitution of manganese by other $3 \mathrm{~d}$ transition metals does not affect the diffuse rings, the same for substitution of titanium by germanium or slicon. Chemical ordering is then ruled out by the experimental evidence. In addition they observed diffuse rings in an approximant crystal. The diffuse rings remain then as a very strong feature of the quasicrystals and related crystals that cannot be accounted for by the chemical disorder and that is rather related to basic topological properties.

Recently, a new understanding of the origin of localized diffuse scattering has been proposed by Newman et al. [16]. It is based on the construction of quasicrystals and approximant crystals using the canonical cell model developed by Henley [17]. The canonical cell model provides a very general framework for cluster based structure. Therefore, this approach of 
diffuse scattering is attractive since most of the difficulties of other models are avoided. It is able to naturally generate the disorder by considering all the allowed configurations. Such a complete exploration of configuration is beyond the possibility of an cosahedral glass model.

In icosahedral glass model, atomic decoration imposing more or less restrictions can always be adapted to reproduce particular observations. The interest of the canonical cell model applied to diffuse scattering is to point out the averaging effect. The diffraction patterns simulated by Newman et al. using the canonical cell model suggests that the observations should depend on the probe size and diffuse rings may have a fine structure. Up to now, no fine structure and average effect has been observed on the diffuse rings. The present work reports a comparative study of X-ray and electron diffraction patterns carried out on $\mathrm{Al}-\mathrm{Li}-\mathrm{Cu}$ quasicrystals coming from the same ingot. While the diffuse rings observed on X-ray diffraction pattern are structureless, we have observed a fine structure on the electron diffraction patterns.

\section{Experimental Conditions}

It is worth noting that the X-ray and electron diffraction patterns must be performed on samples of the same origin because the $\mathrm{Al}-\mathrm{Li}$-Cu quasicrystal structure is very sensitive to heat treatment and chemical composition. In our first attempt of studying the diffuse scattering, we used as-cast Al-Li-Cu quasicrystal ingots. In that case, diffuse rings were absent and diffuse scattering consists in more or less pronounced streaks that we analyzed in details [18]. We have then studied, by transmission electron microscopy, samples coming from the ingot in which the large triacontahedral shaped Al-Li-Cu quasicrystal have been taken out for the X-ray precession patterns [8].

The Al-Li-Cu quasicrystals were prepared by the Péchiney Research Centre owing to a dedicated elaboration process [19]. Bulk quasicrystal samples showing dendrites with a triacontahedral morphology were obtained. These triacontahedra were typically 100 micron diameter size which allows for exploration of the reciprocal space by X-ray diffraction using the monochromatic Laue and precession techniques.

For TEM observations, slices cut from the bulk materıal were mechanically polıshed down to a thickness of about 150 microns. Subsequently, the samples were electropolished in a doublejet electropolishing device $\left(T=-35^{\circ} \mathrm{C}\right.$, electrolyte: $1 / 3$ nitric acid, $2 / 3$ methanol $)$. Samples are centered in the electropolishing device in order to thin the part showing the dendritic morphology. TEM observations were made on a CM20 Philips operating at $200 \mathrm{keV}$. Electron Diffraction Patterns (EDP) were taken in the selected area mode. To observe the diffuse scattering, long exposure must be performed (up to $100 \mathrm{~s}$ ), the condensor lenses being fully defocussed.

\section{Diffraction Patterns}

2.1. X-Ray Precession and Laue Patterns. - The X-ray precession and Laue patterns have already been reported in details in a previous work [8]. These precession patterns have been the first ones to be performed on a monodomain quasicrystal. The more exciting feature was the existence of noticeable diffuse scattering rings on the Laue and precession patterns (Figs. 1a, b). The X-ray precession photographs taken along several axes reveals that the diffuse rings corresponds to diffuse spherical shells in the reciprocal space. The rungs are observed on the diffraction pattern taken with a precession axis parallel to a 2 -fold icosahedral axis (Fig. 1a). The most intense rings are located on 5-fold axis and centered on positions which correspond to the I-type reciprocal lattice. The diffuse ring in Figure 1a is centered on a

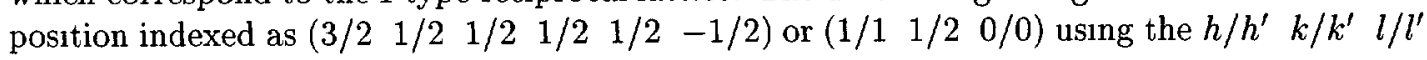




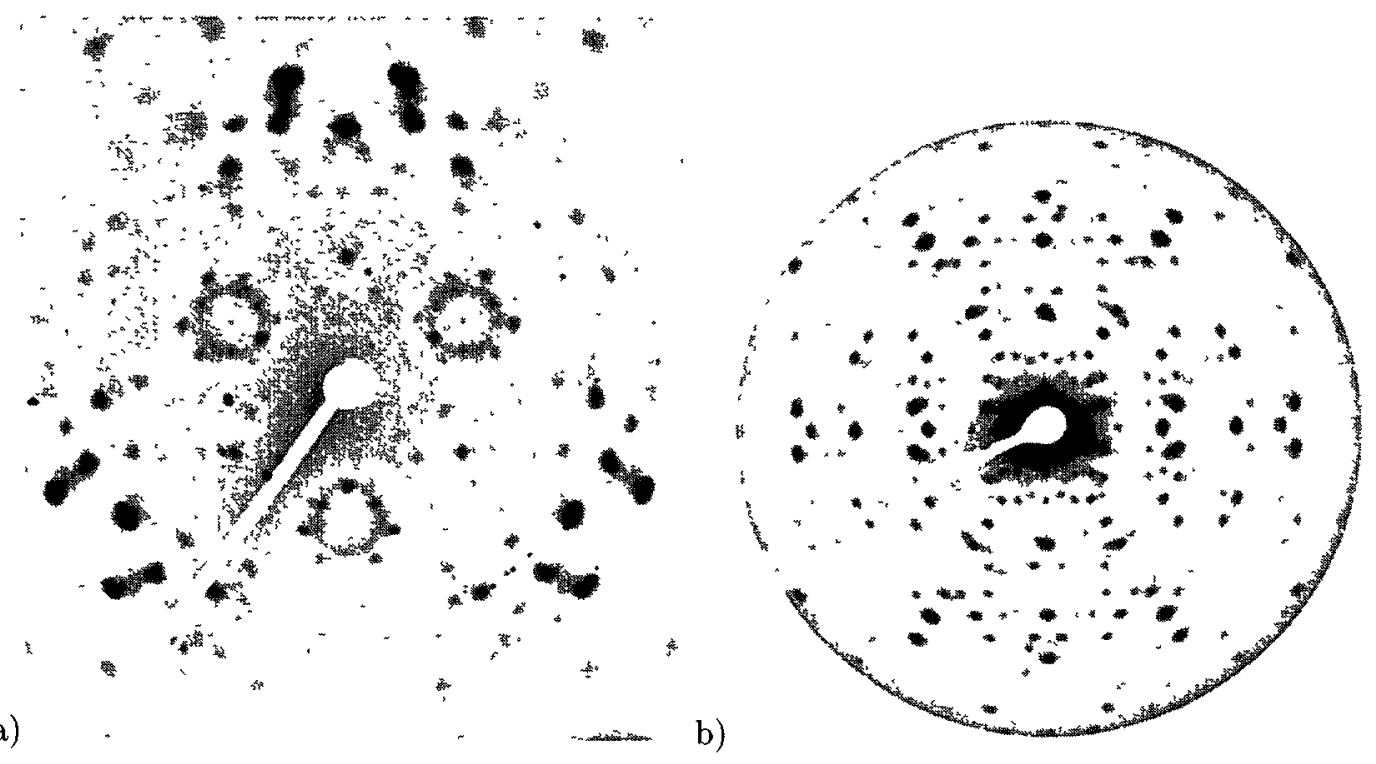

Fig. 1. - a) Monochromatic (MoK $\alpha$ radiation $\lambda=0.711 \AA$ ) X-ray diffraction pattern obtained from the zero-level reciprocal plane with a 2-fold axis parallel to the precession axis. b) Monochromatic transmission X-ray Laue diffraction pattern obtained with a 3-fold axıs parallel to the X-ray beam (CuK $\alpha$ radiation $\lambda=1.54 \AA$ ). The diffuse rings correspond to the intersection of the Ewald sphere with three of the 12 spherical shells located at $|Q|=0.26 \AA$.

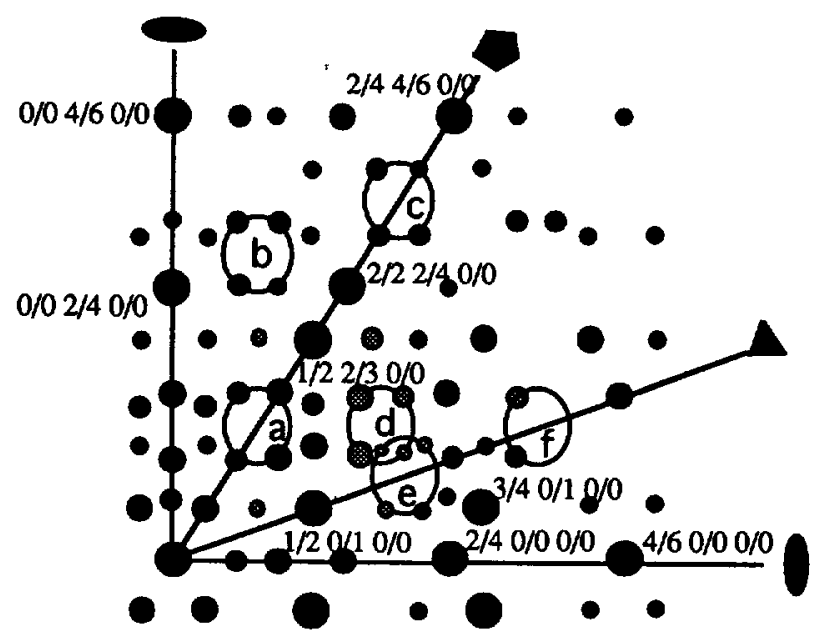

Fig. 2. - Tracing of the 2-fold icosahedral diffraction pattern. The diffuse rings are labelled a, b, $c, d$, e and $f$. All of them are observed on the electron diffraction patterns (see Figs. 3a and 4a). Rings labelled $\mathrm{a}, \mathrm{b}$ and $\mathrm{c}$ are present on the X-ray diffraction patterns (only the rings a are observable in Fig. 1a). The black spots are present on X-ray as well as electron diffraction patterns while the grey ones correspond to spots appear only on the electron diffraction patterns. The diffuse rings are centered on the following positions a: $1 / 1 \quad 1 / 2 \quad 0 / 0 ;$ b: $2 / 3 \quad 3 / 5 \quad 0 / 0 ; \mathrm{c}: 1 / 1 \quad 3 / 4 \quad 0 / 0 ; \mathrm{d}: 2 / 3 \quad 1 / 3 \quad 0 / 0$; e: $1 / 1 \quad 1 / 3 \quad 0 / 0 ;$ f: $2 / 3 \quad 1 / 3 \quad 2 / 2$. 

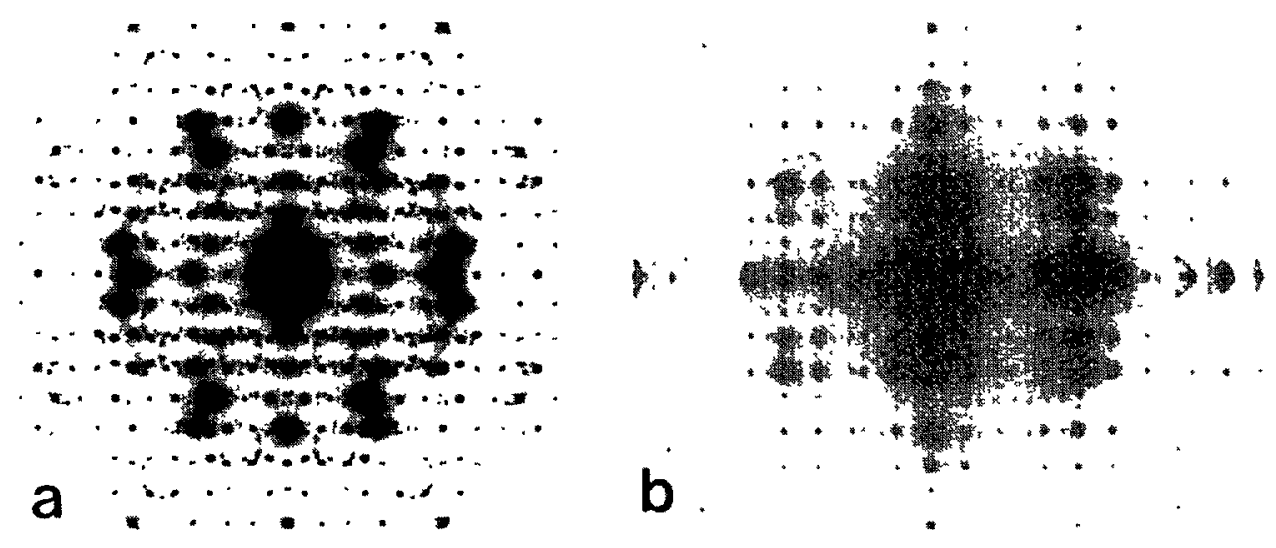

Fig. 3. - Electron diffraction pattern taken along a 2-fold icosahedral axis (Fig. 3a) and along a $<\tau^{2} \tau^{3} \tau^{2}+1>$ zone axis (Fig. $3 \mathrm{~b}$ ). Note the ponctuated rings along the 5 -fold and 3 -fold directions. Figure 3a displays 3-fold and 5-fold direction as indicated in Figure 2, the $\left\langle\tau^{2} \tau^{3} \tau^{2}+1\right\rangle$ axis pattern displays a 5 -fold direction and perpendicularly a 2 -fold direction.

system. Similar diffuse rings but with a much lower intensity were also observed at larger distances from the origin in the reciprocal space: they are centered on positions indexed: $1 / 13 / 40 / 0$ and $2 / 3 \quad 3 / 50 / 0$ (Fig. 2). The diffuse rings shows no fine structure on the X-ray diffraction pattern. The radius of the diffuse shell corresponds to a distance of about $16 \AA$ in the direct space.

Small angle X-ray scattering have been also performed to test whether the diffuse scatterıng was due to a chemical disorder. No diffuse shell centered on the origin of the reciprocal space was detected in small angle scattering experıment. At that step, the X-ray experiments were suggesting that the diffuse scattering was due to a position disorder.

2.2. Electron Diffraction Patterns. - Strikingly, when electron diffraction is performed on samples of the same origin as the ones studied by X-rays, we do not obtained systematically diffuse rings. When rings are present, they are more numerous than on the $\mathrm{X}$-ray diffraction patterns. Figures 3 and 4 displays several diffraction patterns taken along a 2-fold icosahedral axis and $\mathrm{a}<\tau^{2} \tau^{3} \tau^{2}+1>$ zone axis in different regions coming from the same bulk material. Three of them are centered on the positions indicated by the $\mathrm{X}$ ray experiment but other rings are observed on positions centered on a 3-fold diffraction row $\left(\begin{array}{llll}1 / 1 & 1 / 2 & 2 / 2 & \text { and } \\ 2 / 3 & 1 / 3 & 2 / 2\end{array}\right)$ (Fig. 2).

Actually the main difference between $\mathrm{X}$-ray and electron diffraction patterns is that the rings exhibit a fine structure. Figure 5 displays enlargements from Figures 3 and 4 . The rings are constituted by extra diffraction spots which are also observable in other parts of the diagram and form a kind of fine mesh grid. Comparison between Figures $3 a, b$ and $4 a$, b shows that the details of the fine structure vary from one region to the other while some features are preserved. The most intense spots are indexable according to an icosahedral phase as for the Xray diffraction patterns. There are spots indexable as quasicrystal spots which are common to all the diagrams and are undistorted. The weakest undeformed spots are indexed $3 / 4 \quad 0 / 1 \quad 0 / 0$ or 26,41 with the N, M notations which corresponds to $Q_{\text {perp }}=0.48$. On the contrary, the spots of higher $Q_{\text {perp }}$ component forms the variable network. They exhibit deviation to the 1deal cosahedral positions and have strongly anisotropic shapes. Though the spots seems to form a fine mesh net, we did not find a reliable period because of the strong structure factor. Still the 


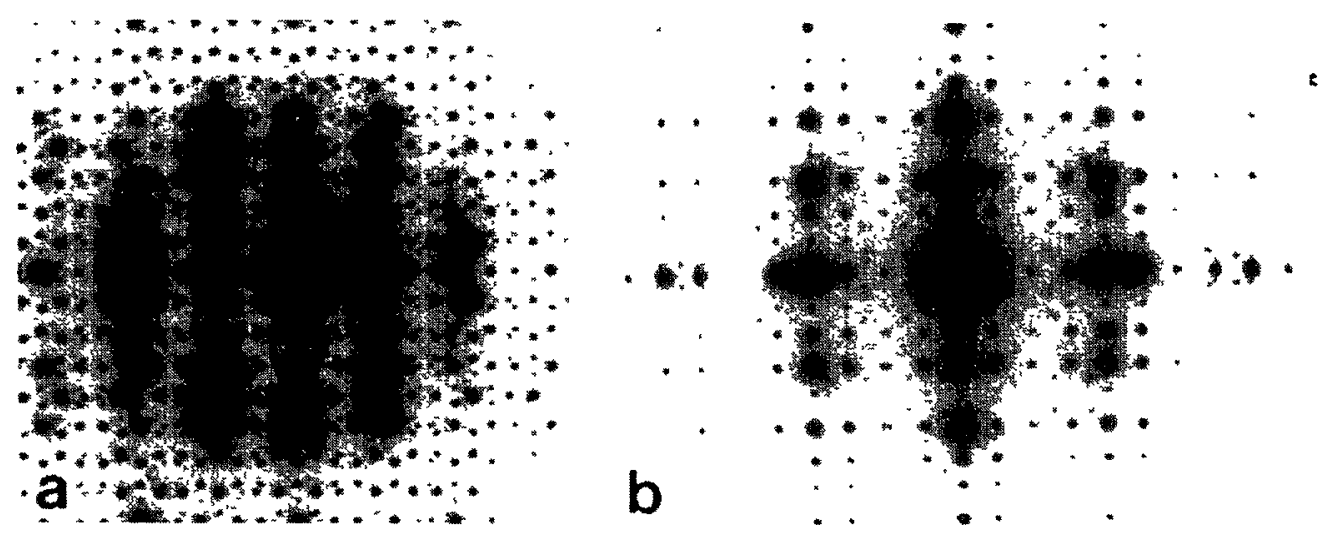

Fig. 4. - These EDPs have been taken in other regions than the EDPS in Figure 3. In both cases, the rings are still ponctuated but they show a fine structure different from Figure 3.
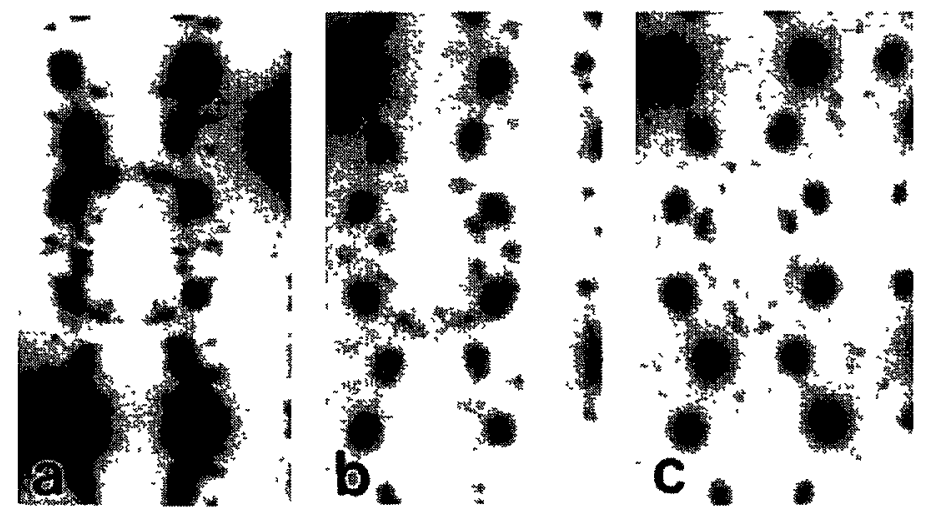

Fig. 5. - Enlargements of the ponctuated rings. Though the average shape of the ring is preserved, the fine structure is quite different.

distance between the closest spots indicates that, if the underlying structure is a crystalline approximant, its cell parameters should be larger than $30 \AA$. However, an approximant crystal structure would not be sufficient to generate all the details observed on the diffraction patterns. In particular, the anisotropic shapes of the extra spots are not accounted for by an approximant crystal and require to introduce a strain distribution. Actually, it does not seem here very important to find an indexation for all the type of diffraction patterns. The important point is the diversity of fine structure within a millimeter size sample. From the same ingot giving the $\mathrm{X}$-ray precession pattern shown in Figure 2, we have obtained EDPs showing different types of ponctuated rings and sometimes we also get EDPs (Fig. 6) without ponctuated rings but diffuse streaks analogous to the ones found in the as-cast Al-Li-Cu quasicrystals [18].

\section{Discussion and Conclusion}

The difference between X-ray and electron diffraction, apart from the atomic structure factor, is essentially the size of the diffracting volume. In the present study, the diffracting volume investigated by X-rays is approximatly $(100 \mu \mathrm{m})^{3}$. In comparison the diffracting volume for 


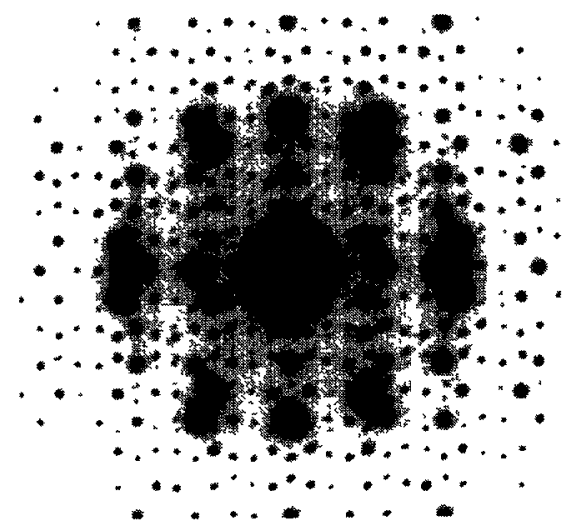

Fig. 6. - Some regions display diffuse streaks instead of rings. These streaks are perpendicular to the 2-fold and 5-fold direction. The most intense diffuse streaks are located between the two intense spots on the 5 -fold directions.

electron diffraction is very small since EDPs are taken on selected area of $0.5 \mu \mathrm{m}$ diameter for a sample less than $50 \mathrm{~nm}$ thick. The ratio between the diffracting volumes is then larger than $10^{7}$. It is then natural to consider that the absence of structure on the rings on X-rays diffraction patterns is due to an average effect. This is confirmed by the fact that the EDPs also show that the structure is not homogeneous at the submicronic scale.

Though it should be in principle always possible to find an adequate decoration of the icosahedral glass to describe the different fine structure of the diffraction patterns, a different point of view, i.e. canonical cell tilings, seems here well adapted. The observations of fine structure and featureless rings shows a strong analogy with the diffraction patterns simulated by Newman et al. [16] using variants of canonical cell tilings. The so-called canonical cell model is dedicated to the construction of realistic tiling which avoid the main problem of usual cluster description: that is to say specifying cluster and glue atoms. The systematic investigation of the cell geometry leads to the identification of canonical configuration related to the possible linkage consıstent with icosahedral cluster structure. Newman et al. have applied this model to approximant crystal. They have build an algorithm giving all the possible canonical cell tilings corresponding to a particular rational approximant. When the average is taken on all the possible structures for a given approximant structures, diffuse scattering rings are generated on the average diffraction patterns.

Newmann et al. have simulated averaged diffraction patterns considering all the types of $5 / 3$ approximant crystal. They predict diffuse rings centered on $1 / 1 \quad 1 / 2 \quad 0 / 0$ and $1 / 1 \quad 1 / 2 \quad 2 / 2$, positions which respectively belong to a 5 -fold and 3 -fold icosahedral axis. The rings observed by TEM are then in agreement. It is worth noting that icosahedral glass model are also able to account for diffuse rings at these positions. The main difference with Newmann et al. is that the question of average structure is addressed in their work. The present observations of ring structures depending on the scale of investigation supports the idea that averaging is responsible for the occurence of diffuse rings.

The description in terms of a mixture of all the possible tilings derived from the canonical cell conditions provides indeed a very realistic picture for the microstructure examplified by the Al$\mathrm{Li}-\mathrm{Cu}$ icosahedral phase. Numerous TEM studies have shown that the Al-Li-Cu quasicrystals display numerous deviations to perfect icosahedral symmetry. Earlier works on this system mentioned important peak broadening and shifts [20]. Then high order approximant crystals 
have been identified by electron diffraction but these phases were never met in the $\mathrm{Al}-\mathrm{Li}-\mathrm{Cu}$ phase diagram [21]. More recently modulated structures have also been observed on high resolution images [22]. Additional intensities and spot deviation were also reported [23]. All these observations are not really in contradiction with each other and they examplified all the different types of disorder or microstructure that can exıst in a quasicrystal related structure. However, such a diversity also proves that a realistic description of quasicrystals, and at least of the Al-Li-Cu phase, must explore all these possibilities. The model proposed by Newman et al. have here the great advantage to be adaptable to all the local scale deviations and also for the more global aspects.

However, as we observed that there are common spots which are indexable as quasicrystal ones up to a certain $Q_{\text {perp }}$ component, it seems still valid to consider the Al-Li-Cu phase as globally quasicrystalline. It is possible that a perfect $\mathrm{Al}-\mathrm{Li}-\mathrm{Cu}$ quasicrystal exists but the degree of perfection strongly depend from the scale of investigation.

The present study gives a good example for apparent contradiction between X-ray and electron diffraction experiments which might occur in quasicrystals and related phases because of the intrinsic proximity and coherency of these structures. It follows that one must be very careful in the comparison. The comparison might be even more delicate between high resolution electron microscopy images and X-ray diffraction.

\section{Acknowledgments}

The authors are grateful to Drs. Sainfort and Dubost from the Péchiney Research Centre for providing the quasicrystalline samples.

\section{References}

[1] Sainfort P. and Dubost B., J. Phys. Colloq. France 47 (1986) C3-321.

[2] Tsal A.P., Inoue A., Yokoyama Y., Masumoto T., Mater. Trans. Jpn Inst. Met. 31 (1990) 98.

[3] De Boissieu M., Durand-Charre M., Bastie P., Carabelli A., Boudard M., Bessière M., Lefebvre S., Janot Ch. and Audier M., Philos. Mag. Lett. 65 (1992) 3-147.

[4] Bessière M., Quivy A., Lefebvre S., Devaud-Rzepski J. and Calvayrac Y., J. Phys. I France 1 (1991) 1823-1836.

[5] Friedel J. and Dénoyer F., C.R. Acad. Scr. Paris. II (1987) 171-174.

[6] Henley C.L., Quasicrystals, the state of the art, Directions in Condensed Matter, Vol 11, D.P. Di Vincenzo and P.J. Steinhardt Eds. (1991) pp. 429-524.

[7] Mukhopadhay N.K., Ranganathan S. and Chattopadhyay K., Phılos. Mag. Lett. 56 (1987) 121.

[8] Dénoyer F., Heger G., Lambert M., Lang J.M. and Sainfort P., J. Phys. France 48 (1987) 1357-1361.

[9] Goldmann A.I., Guryan C.A., Stephens P.W., Parsey J.M., Aeppli G., Chen H.S. and Gayle F.W., Phys. Rev. Lett. 61 (1988) 1962-1965.

[10] Swamy V.T., Ranganathan S. and Chattopadhyay K., J. Mater. Res. 4 (1989) 539.

[11] Gibbons P.C., Kelton K.F., Levine L.E. and Phillips R.B., Philos. Mag. B. 59 (1989) 593-618.

[12] Gibbons P.C. and Kelton K.F., Philos. Mag. B. 66 (1992) 639-651. 
[13] Mukhopadhay N.K., Chattopadhyay K. and Ranganathan S., to be published in Bull. Mater. Sci.

[14] Gibbons P.C. and Kelton K.F., J. Non-Cryst. Sol. 153-154 (1993) 165-170.

[15] De Ridder R., Van Tendeloo G. and Amelinckx S., Acta Cryst. 32 (1976) 216.

[16] Newman M.E.J., Henley C.L. and Oxborrow M., Philos. Mag. B 71 (1995) 991-1013.

[17] Henley C., Phys. Rev. B 43 (1991) 993-1020.

[18] Proult A., Donnadieu P., Wang K. and Garoche P., J. Phys. I France 5 (1995) 1615-1624.

[19] Dubost B., Lang J.M., Tanaka M., Sainfort P. and Audier M., Nature 324 (1986) 48.

[20] Yu N., Portier R., Yu-Zhang K. and Bigot J., Philos. Mag. Lett. 57 (1988) 35-39.

[21] Knowles K.M., Proceedings of the I.L.L./CODEST Workshop, Quasicrystalline materials, Grenoble, Ch. Janot and J.M.Dubois Eds. (Singapore: World Scientific) (1988) 159.

[22] Staiger W., Yu D.P. and Kléman M., Philos. Mag. A. 67 (1993) 991-1005.

[23] Nissen H.U. and Beel1 C., J. Non-Cryst. Solids 153-154 (1993) 69. 